\title{
Meta-Ethnography as a Tool for Synthesis Qualitative Research in Health
}

\author{
Ahmad Kalateh Sadati ${ }^{1, *}$ \\ ${ }^{1}$ Department of social sciences, Yazd University, Yazd, Iran \\ ${ }^{*}$ Corresponding author: Ahmad Kalateh Sadati, Department of Social Sciences, Yazd University, University Blvd, Safayieh, P.O. Box: 89195741, Yazd, Iran. Tel: +98-3531232222, E-mail: \\ asadati@yazd.ac.ir
}

Received: 22 Sep 2016

Accepted: 21 Nov 2016

Ppub: 23 Dec 2016

\begin{abstract}
Background: The aim of this study was introduce a qualitative approach for synthesis previous findings in health called metethnography. Generally, qualitative meta-ethnography is a study design used to systematically assess previous research studies to derive new concepts constructions about the subject. Epistemologically, this is a meta-interpretation about past studies.

Methods: Noblit and Hare (1988) proposed a meta-ethnography method which includes seven stages: (1) Getting started involves formulating a research idea that is 'worthy of the synthesis effort'. (2) Deciding what is relevant. (3) Reading the studies involves careful attention to the conceptual content of the included studies. (4) Determining how studies are related involves identifying and describing the 'metaphors' or concepts in studies and 'translating' or comparing them to those in other studies. (5) Translating studies into each other involves sorting concepts from primary studies into conceptual categories; (6) Synthesizing translations involves developing a model that helps to make sense of the topic under investigation. (7) Expressing the synthesis involves output and dissemination of findings.

Results: For quality appraisal using of structured checklists is proposed. However, adequate description for 'well enough' methodologically report is the main goal.

Conclusions: Generally, this study involves identifying ideas from the studies included and progressively abstracting these ideas into a line of argument, or conceptual model can help for providing thick description about different issues in health.

Keywords: Health System; Meta-Ethnography; Qualitative Research; Health
\end{abstract}

